\title{
Exploring the decision to participate in the National Health Service Bowel Cancer Screening Programme
}

\author{
Merryn Ekberg; Matthew Callender; Holly Hamer and Stephen Rogers
}

Cancer is a leading cause of mortality and one of the most feared diseases in modern society. A combination of early detection, accurate diagnosis and effective treatment provides the best defence against cancer morbidity; therefore, promoting cancer awareness and encouraging cancer screening is a priority in any comprehensive cancer control policy. Colorectal cancer is the third most common form of cancer in the UK and in an effort to reduce the high incidence, prevalence, morbidity and mortality rates, the National Health Service (NHS) has introduced the NHS Bowel Cancer Screening Programme (NHS BCSP). For the NHS BCSP to succeed in its goal of reducing the incidence and prevalence rates for colorectal cancer, individuals need to be persuaded to complete the test. Since it was first introduced in 2007 , however, participation rates have been low. In an effort to understand why participation rates remain low, this article reports on the findings of a series of focus groups conducted in the East Midlands of England. These focus groups were designed to explore the factors that influence an individual's decision to participate in cancer screening. The findings revealed eight factors that affected participation in the NHS BCSP: (i) the association of screening with entry into old age; (ii) prior experience with health systems; (iii) the support of a significant other;

(iv) individual perceptions of risk (and benefit); ( $v$ ) fear of becoming a cancer patient after the screening test; (vi) lack of disease symptoms; (vii) embarrassment associated with completing the test and (viii) messages that adopt

a paternalistic ethos. Overall, our results suggest that more people may participate in the screening programme if it was more sensitive to these psychosocial and contextual factors that shape an individual's decision to be tested. European Journal of Cancer Prevention 00:000-000 Q 2014 Wolters Kluwer Health | Lippincott Williams \& Wilkins.

\section{Introduction}

Colorectal cancer (CRC) is a leading cause of morbidity and mortality in the western world (Hewitson et al., 2007). In 2010, 40695 people were diagnosed, and B16 000 people died from CRC in the UK (Cancer Research UK, 2010). CRC is the third most common type of cancer in men and women and the second most common cause of cancer mortality in the UK, and it accounts for around $10 \%$ of all cancer deaths (Cancer Research UK, 2010). Late diagnosis is one of the key factors contributing to CRC mortality, and in an effort to reduce the high incidence, prevalence, morbidity and mortality rates, the UK government has implemented the National Health Service Bowel Cancer Screening Programme (NHS BCSP).

The NHS BCSP uses a biannual faecal occult blood test (FOBt) to screen for blood in the stools. Early detection through the FOBt has been shown to reduce mortality from CRC by $16 \%$ (Hewitson et al., 2007). In the UK, people aged 60-69 years are sent an invitation letter, information leaflet and test kit to their home address. Recipients are encouraged to complete the home-based test and then return the samples in the reply-paid envelope. A positive test result (i.e. detection of blood in

the stools) indicates the need for a follow-up diagnostic examination, usually a colonoscopy. To make an informed decision about whether to complete the screening test, individuals are informed that the screening test is not a diagnostic test and that the sensitivity and specificity of the test is not absolute. This means that some individuals who return a positive FOBt will not have cancer, or alternatively, some individuals with early stage CRC will not return a positive FOBt. Despite the occasional false- positive or false-negative result, clinical trials have shown that the FOBt is the safest and most cost-effective screening test currently available (Flight et al., 2008). 
Population-wide cancer screening is one of several programmes that contribute to the national strategy to improve cancer services and reduce cancer morbidity in the UK (Department of Health, 2007). Cancer screening is also emblematic of the ideological shift from the 'old public health' to the 'new public health' (Tulchinsky and Varavikova, 2010). Whereas the 'old public health' was concerned with reducing the burden of cancer after diagnosis, the emphasis in the 'new public health' is on primary prevention, health education and health promotion. In what Armstrong (2012) has described as 'surveillance medicine', individuals are encouraged to seek health information from reliable sources, and on the basis of this information, they are encouraged to make rational and informed choices about how to delay, avert or prevent the onset of illness. The role of government is limited to a 'surveillance' or 'monitoring' function, whereby public health officials use surveillance techniques such as screening to identify individuals at risk. Such characteristics concur with Lupton's (1995) suggestion that within the new public health regime, there is more emphasis on promoting self-efficacy and empower- ing individuals to make informed choices about their health, rather than relying on a paternalistic government to manage the nation's health.

Alongside this transition from the 'old' to the 'new' public health, modern western societies are undergoing a demographic transition. This means that whereas one in six people in the UK are currently aged 65 years and over, by 2050 one in four people will be aged 65 years and over (Cracknell, 2010). As CRC is a disease that primarily affects older people, where eight out of every 10 patients are over 60 years of age (West et al., 2007), it is likely that the annual incidence of CRC will increase as the UK continues to undergo this demographic transition. For many older people, cancer is the most feared disease (Griffiths, 1995) and has been shown to be feared more than having a heart attack, developing Alzheimer's disease or being a victim of a terrorist attack (Department of Health, 2007). Research has shown that this fear of cancer is experienced by individuals with no diagnosis as well as by those who have recovered from cancer. Horlick- Jones (2011), for example, describes how 'fear of cancer recurrence' can have a detrimental effect on the quality of life and wellbeing of people who are in the recovery phase or who have recovered from cancer. It is likely that this 'fear of cancer' is a key factor when older people make the decision about whether or not to participate in CRC screening, and it is important that such fears are sensitively addressed in the NHS BCSP.

For the NHS BCSP to succeed in its goal of reducing the incidence and prevalence rates for CRC, individuals need to be persuaded to complete the test. Since it was first introduced in 2007, however, participation rates have been low, especially for men, people from lower socioeconomic backgrounds and people from ethnic minority groups (Weller et al., 2007; Szczepura et al., 2008). In an effort to explain these differential participation rates, previous studies have shown that a range of social and environ- mental factors can influence the decision to participate in CRC screening programmes. These include a person's sex, age, ethnicity, socioeconomic status, level of education and health literacy (Keighley et al., 2004; Wackerbarth et al., 2005; Thompson et al., 2011). O'Sullivan and Orbell (2004) found that whereas the FOBt was seen as socially acceptable, concerns were raised about collecting and storing faecal matter. Similarly, participants in a study by Chapple et al. (2008) indicated a reluctance to handle stools and send samples in the post for analysis, but suggested that 'civic responsibility' was a driver for completing the test. Other investigators have explored the role of general practitioners (GPs) in an individual's decision-making process, and these studies suggest that uptake would be higher if GP's were more actively involved in promoting the benefits of the BCSP to their patients (Beeker et al., 2000). Damery et al. (2010) conclude from their study that 'the success of population-based screening for CRC will be determined to a large extent by GP attitudes, beliefs and support, particularly with regard to faecal occult blood testing' (West et al., 2007). It is likely that the traditional doctor- patient relationship of trust is still an important factor in a person's decision-making process, and especially for older people who are the primary target for the NHS FOBt. We argue that the uptake, and thereby the success, of the NHS BCSP is largely determined by each individual's response to the programme, and that this response is shaped by their knowledge and attitudes of CRC in particular and public health more broadly. In turn, an individual's response to the BCSP is determined by a combination of biographical, psychosocial 
and contextual factors. This study set out to explore these interlocking factors by investigating the attitudes of individuals living in the East Midlands of England towards the NHS BCSP.

\section{Methods}

A focus group methodology was used to identify and understand the factors that encourage or discourage individuals from participating in the BCSP. The focus group methodology was chosen because we were interested in both individual responses to the questions and interactions that take place between individuals, as each member of the group responded to the discussion questions and to each other (Kitzinger, 1994). We noted whether people confirmed or modified their own response after listening to the views of others.

Approval from a multicentre NHS research ethics committee was obtained to conduct this study. A semistructured interview guide was used during the focus groups, with additional probing questions drawn spontaneously from the discussion (see Fig. 1 for the interview schedule). The interview guide was developed from a preliminary review of the published literature of previous studies that explored the drivers and barriers to participation in cancer screening. A pilot focus group was conducted to evaluate the questions and to appraise the suitability of conducting mixed-sex groups. Given the sensitivity of the topic, we initially planned to conduct single sex focus groups, but after the pilot focus group, we decided that the mixed sex group did not inhibit open discussion, but rather, stimulated an interesting discussion about the gendered differences in attitudes towards the screening programme.

Fig. 1

(1) Are you aware of the NHSBCSP?

(2) Have you seen any posters or leaflets raising awareness of the BCSP?

(3) Do you think screening is important? why or why not?

(4) Can you tell us what you know about the disease cancer?

(5) Can you tell us what you know about bowel cancer?

(6) What might be the benefits of the BCSP?

(7) Have you participated in the BCSP? why or why not?

(8) Doyou have any family or friends that have decided to participate, or not, in the BCSP? have you discussed the reasons why they did or did not participate?

(9) Have you undergone screening for any other cancers?

(10) What changes could be made to the NHS Bowel cancer screening programme to encourage more people to participate?

Focus group questions. NHS BCSP, National Health Service Bowel Cancer Screening Programme.

Including the pilot focus group, we conducted five mixed-sex focus groups. The total number of participants was 33 (15 male and 18 female). These were drawn from three towns within the county that were socio- economically representative of the county as a whole. Community-based public health staff with links to community groups used purposeful sampling to recruit a balanced mix of ages, sex and socioeconomic back- grounds for people who met the eligible criteria for participation. Exclusion criteria for the study were people under 60 or over 69 years of age, people living outside the county and those who were non-English speaking. As all our participants were aged 60 years and over, $85 \%$ were retired ( $94 \%$ of women and $73 \%$ of men). Previous 
occupations, however, included a self-employed window cleaner, shop assistant, secretary, government officer and engineer; thus, our sample included a wide range of occupational backgrounds. A brief postcode analysis indicated a good distribution across neighbourhood deprivation quintiles, with a mean index of multiple deprivation score of 22.06 and a deprivation quintile of 3 .

Upon arrival, participants were welcomed by the research team and offered light refreshments. Being a sensitive topic, we wanted to ensure that all the participants were comfortable talking as a group. As a prelude to the focus group discussion, a short presentation on the epidemiology and risk factors for bowel cancer was given to set the context for the study and to initiate thinking around the topic. The participants were then provided with a participant information sheet and were invited to sign a consent form that provided an assurance that their participation was voluntary, that they were not obliged to answer all questions, that they were free to withdraw from the study at any stage and that their privacy and confidentiality would be protected throughout the transcription, analysis and write up phases of the study.

The focus groups were constructed specifically for the research, rather than being a pre-existing or natural group. With a few exceptions, group members did not know each other before the focus group session. We felt this would enable a more open and honest discussion because the participants would be less fearful about disclosing personal or embarrassing information to friends or colleagues. For consistency, all the focus groups were facilitated by the same researchers. All participants were aged between 60 and 69 years because the FOBt is offered only to this age group. Two focus groups were held during the day and three were held in the evening. This was a deliberate strategy to make the focus groups accessible to a wider range of people. The duration of each focus group was between 60 and $90 \mathrm{~min}$. Data collection was continued until data saturation, that is, until we felt that no new ideas were emerging from the discussions. Participants were given a $£ 20.00$ store voucher at the end as an expression of gratitude for their contribution. They were unaware of this at the recruitment stage, thereby ensuring that altruism, rather than payment, was the reason for participating.

After transcription, the transcripts were analysed to identify the factors that influenced the decisions to be, or not to be, tested for CRC. In presenting the quotations, we have indicated whether it is a male (M) or a female (F) speaking.

\section{Findings}

From the analysis of our qualitative focus group data, we identified eight factors that influence the decision to participate in the BCSP. These were a person's age and wider social attitudes to ageing; a person's previous exposure to healthcare and prior experience with health screening; the influence of significant others; an individual's perception of risk and their propensity to display an optimistic bias; a general fear of cancer and attitude of 'ignorance is bliss'; lack of disease symptoms resulting in an attitude of not needing to be tested; embarrassment and finally, a resistance to paternalistic healthcare. Ultimately, what emerges is a complex interplay between individuals and the wider healthcare system as individuals attempt to balance the risks and benefits as they decide whether or not to complete the screening test.

\section{Association of screening with entry into 'old age'}

The first factor to influence the decision to participate in CRC screening was a person's age. Our participants appeared to associate the screening test with entry into the socially constructed category of 'old age' and wanted to avoid the stereotypical image that associates older age with chronic illness. They resisted the idea that once a person reaches the age of 60 years, their health declines and they become a perpetual patient, in this case a 'cancer patient'. From the point of view of our participants, these negative images of age and illness were amplified when they received a letter from the NHS saying that they were now expected to complete a bowel cancer screening test. One of our participants received his letter on his 60th birthday and felt this was stepping onto the slippery slope. He exclaimed: 
Whereas this participant saw age as the 'slippery slope', others saw turning 60 as a social stigma or as having 'one foot in the grave'. In a dialogue between a male and a female participant, the following was discussed:

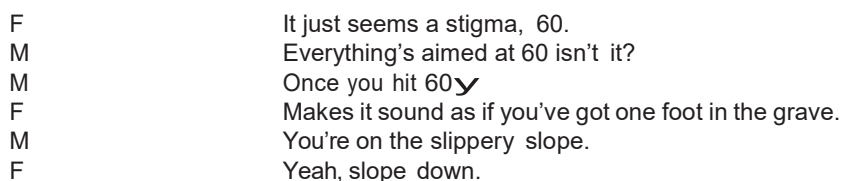

\section{Exposure to health screening}

The second factor to influence participation was prior exposure to, and past experience with, health screening. Our participants suggested that more frequent exposure to health screening is likely to result in an increase in body awareness and a greater acceptability of medical screening. This implies that prior events in an individual's life (especially events that bring that person into contact with healthcare services) can be a powerful influence in shaping that individual's response to health screening. Our participants further suggested that women were more likely to participate in the BCSP than men because many women (particularly women who have been through pregnancy and childbirth) have had more experience of health screening, and therefore, were less likely to be embarrassed when invited to complete the FOBt. In one exchange between male and female participants, we found that both men and women agreed that women are more likely to participate.

\footnotetext{
F I think women will [participate] because women have had the breast checks, they have smear tests, they've had kids most of them and you know, there's no dignity at all when you've had a child so l just think they're more aware of their bodyY. Men are a bit more wary and personally I don't think men would do it as much.

F I think women [are more likely to participate in CRC screening] because I think we're more bodily aware.

M Welly Yes, it possibly is women, $Y$. I think perhaps women do get checked over more often for other things and so this is one thing that they'd be quite happy to have done as well.
}

\section{Significant others}

The third factor to influence the screening decision was the presence or the absence of support and encouragement from significant others. Being in a significant relationship with a husband, wife or partner can be a positive driver for FOBt participation, especially for married men. This has been shown previously in a study by Thompson et al. (2011), who concluded that the best way to target men is through their wives. In contrast to Thompson et al. (2011), our study indicates that targeting people in relationships can be beneficial for both men and women. As the FOBt is completed by individuals in the privacy of their own home, without a face-to-face consultation with a healthcare professional, it appears that having a husband, wife or partner may lower an individual's anxiety about the test. Furthermore, being in a significant social relationship may increase the relative value and motivation to complete the FOBt. One of our married male participants, who had completed the screening test, acknowledged the encouragement he had received from his wife when he said:

M I think if I was on my own l'd have probably just binned it.

Similarly, one of our female participants suggested that married men would be more likely to complete the test than single men. In her words:

F I think men with partners are probably more likely to fill it in, because they'd probably get nagged by their wives.

Two women, however, explained that it was the presence of a significant other that encouraged people to complete the screen, and that this could be important for both men and women. In 


\section{their words:}

F I think it does work if you have either a wife or a partner, if there are two of you in the situation, I can understand one person on their own would probably just say 'I can't be bothered'.

$\mathrm{F}$ I tend to think if you're married that you would make sure you each did it. I'm not saying you'd be put off if you live on your own but I think if you're married and you both got it through, I mean I know it doesn't always work but generally you would, if you're going to do it you'd make sure your husband did.

\section{Perception of risk}

Perception of risk was the fourth factor to influence participation, and our participants suggested that one of the reasons for not completing the FOBt was the belief that 'it [cancer] won't happen to me'. In doing so, they are referring to the 'optimistic bias', a term used to describe people underestimating the risk of something occurring and when they hold a belief that bad events happen only to other people (Lupton, 1999; Slovic, 2000). The participants in this study were found to underestimate the risk of developing CRC and admitted that they felt cancer would not affect them. As one gentleman explained:

M It's what I cally the mobile phone syndrome it's the 'it won't happen to me so why bother?' $Y$ people think 'well I won't have a car crash' but then they've had a car crash.

Although most of our participants believed that cancer affects only other people, their perceived risk of developing $\mathrm{CRC}$, and hence the likelihood of them completing the FOBt, was shaped by key events in their unique biographical past. One participant, for example, described how her late husband was diagnosed with cancer despite having lived a healthy life, and after his death, her decisions about health screening continue to be shaped by this event. In her words:

F Yyou think cancer is what somebody else gets, not what you get $y$ then, l've got my husband who ran marathons, did cycling, didn't smoke, occasionally dranky and then something like that happens [cancer diagnosis] and in so many months he passed away, and so straight away my attitude was 'blow this healthy living $y^{\prime}$ '. So, you know when people tell you [about cancer screening], again [I think that] it's not going to happen to me' but then you don't know do you? If it's going to happen it's going to happen, but do I want to know any earlier? I know it could save my life, buty

In contrast, some participants described how knowledge of another individual who had been diagnosed with cancer increased their willingness to participate in health screening by increasing their perceived vulnerability and reducing their optimistic bias. Importantly, a person's subjective assessment of risk will often be influenced by their biographical past, suggesting that a key strategy for health promotion is to reduce the gap between actual and perceived risk. As one woman explained:

F If somebody you know has suffered with whatever then it makes you think 'should I be tested for this, should I do that?'

\section{Fear of cancer}

'Fear of cancer' was the fifth factor to influence the decision to be screened. Our participants described cancer as the major health fear of their generation. They suggested that individuals may not complete the FOBt due to an underlying 'fear of the result'. Although this fear was felt by both men and women, it was more readily expressed by men. Such behavioural responses to the FOBt are in opposition to the new public health ethos, where individuals are encouraged to be more active in seeking health information and more responsible in acting on that information (Lupton, 1999). Our participants suggested that some people prefer to remain 'ignorant' rather than seek knowledge that they could have cancer. In one dialogue, the participants explained:

M I do feel today, in today's society, the phrase 'the big C', as it's euphemistically called, frightens lots of peopley

F Yes.

M And it tends to affect the whole of society, but I don't think there's anybody of my age now who hasn't heard, or knows of somebody who has had contact with somebody who's had cancer. Looking back to my mother and father's time, the great killer was, $y$ tuberculosis, and that died away and then cancer came along and it makes you wonder what nature's going to throw at the human race next in the future. 
Some recognized 'fear of cancer' as a reason to be tested rather than a reason not to be tested, whereas others recognized the paradox between understanding the benefits but not wanting to know the result. In one exchange, the participants said:

F It doesn't make sense [non-participation in CRC screening] if everybody is so scared about it why don't they do something about it?

$\mathrm{M}$ Yeah, but ignorance is bliss, you know. M Especially amongst men.

M That's true though, yeah, it's true.

\section{Lack of symptoms}

'Lack of symptoms' was the sixth factor to influence the decision to participate in the BCSP. This is especially problematic because many individuals will not display or notice symptoms in the early stage of the disease. This further compounds the effect of the optimistic bias because the importance of the FOBt is weakened when the perceived risk of developing cancer is low. This may be a particularly strong barrier for older people who are more familiar with the traditional model of consulting a doctor only when symptoms appear. One participant told us that people do not complete the testy

F Because if you've got no symptoms, you don't feel unwelly then you don't think about it.

This was echoed by another participant who said:

F So the issue you have really with doing any sort of test like that is you [have a symptom] which you then have to decide what to do withy Yeah, so you obviously need to be going to the doctor with a complaint of some sort to be tested.

\section{Embarrassment}

'Embarrassment' was the seventh factor to influence the decision to participate in the BCSP, and our participants suggested that the FOBt (and CRC more broadly) was something they were embarrassed to discuss with others, even in the privacy of their own home. This was seen as a challenge to be addressed in health promotion campaigns, and failure to address the sensitive nature of the screening test could result in both a low participation rate and a low adherence rate. Individuals may agree with the concept of screening, and may understand that the benefits of screening outweigh the risks, but at the same time, they may find the practical realities of completing the test to be a major obstacle. Thus, the programme may have a lower adherence rate than would be possible if the 'embarrassment factor' could be overcome. Among our participants, there was a general consensus that embarrassment, along with threats to dignity and privacy, are major barriers to their own personal decision to participate in, and adhere to, the programme. Indeed, our findings indicate that conversations about CRC among family and friends are often very limited, and thus, the decision to complete the FOBt becomes a very private and personal decision. When asked what prevented people from completing the screening test, one male participant simply replied 'Embarrassment. That's what it is'.

This helps to explain the sex differential in both the participation rate and the adherence rate because in general, men are more reluctant to talk about embarrassing body parts than women. Encouraging people (men and women) to talk about bowel cancer is challenging because it is generally regarded as a socially unacceptable topic for conversation. Our participants captured this in the following dialogue:

\footnotetext{
F Yeah, but if you were out with families or friends for an evening it's not a subject that anybody would ever talk abouty You talk about the weather, you talk about holidays, you talk about families, you don't talk about illnesses.

M No, no.

F I mean even if you saw it advertised on the television it's not the type of thing that you would discuss generally.
} 
participants suggested that the implementation of the new preventative approach to healthcare, where people are encouraged to recognize early symptoms and take measures to prevent illness, tends to alienate or dehumanize their engagement with the health system. Some of our participants associated the messages of preventative healthcare with the 'nanny state', which they interpreted as being a threat to individual freedom and autonomy and as being overly broad and repetitive. This resistance to paternalism was more prevalent among the male participants; for example, one gentleman explained:

M There is a contention which we all hear about in today's society that the state, it's a nanny state, OK? They tell us, don'tsmoke, cut down on your drinking don't become obesey

M Take regular exercise, blah di blah di blahy andy then you're exposed probably unwillingly or unknowingly to a cancer causing agent which changes the DNA within your body $Y$ and it runs amok, and all sorts of cancers can emanate from that one, Imean asbestos forinstance, one fibre of asbestos, I should imagine anybody of my age has been exposed to severaly hundreds in fact. But the contention that it is becoming a nanny state, the amount of money that is actually being spent on media coverage of you should do this, you should do that, and all the rest of it, in some cases could be seen as counterproductive. All this, there's too much nannying going on, you know, really.

\section{Discussion and conclusion}

This study explored the attitudes of people in the East Midlands of England towards the NHS BCSP and identified some of the factors that influence the uptake of the screening test. Much emphasis is placed on 'public and patient choice' within the 'new' NHS, and this is an important part of the context in which the current CRC screening is located (Rowe and Calnan, 2006). This may be seen as contradictory to the idea of 'civic responsibility', which was identified as an emerging theme in a study by Chapple et al. (2008). However, we suggest that 'public and patient choice' and 'civic responsibility' are

compatible concepts as the public can choose to take on board the idea of 'civic responsibility' and, with this, complete the test for their own benefit and for the benefit of society.

What is unique about this study is that we have shown how an individual's biographical past can influence how they respond to the NHS BCSP and how they interpret the messages that encourage participation in the screening programme. In particular, our findings have shown that many individuals perceive the invitation to participate in the BCSP as being associated with entry into old age, and this conflicts with how they view themselves as individuals. While the rationale for targeting the BCSP at people aged 60-69 years is based on age-adjusted incidence rates for $\mathrm{CRC}$, the test represented a 'slippery slope' for participants, whereby they had now entered into a social category that is more vulnerable to chronic illness. The findings also demonstrated how an individual's previous experience with health screening can influence their attitude towards participation in the programme. Previous experiences of health screening, or intersections between one's biographical past and cancer (either personal experience or knowledge of a social contact who had experienced a form of cancer), were seen as important factors that contribute towards shaping the higher value given to messages associated with the BCSP. Finally, our findings suggest that having stable and supportive relationships with significant others can be a positive driver for uptake.

To conclude, we argue that to increase uptake in the NHS BCSP, more attention should be paid to increasing the level of trust between the individual and the national health system. Although the ethos of the 'new public health' is founded on health prevention, health education and health promotion, the participants in this study viewed these messages as a form of medical paternalism, which lowered their weight and value when deciding whether or not to participate in the programme. We suggest further exploration into the interplay between an individual's self-identity and their perceptions of risk in relation to developing bowel cancer. In so doing, future public health initiatives and interventions should be developed that will be more attentive and sensitive to the highly personalized issues surrounding the decision to participate in the NHS BCSP.

\section{Limitations}


The results of this study are limited to a small, self-selecting sample and a small geographical region in the East Midlands of England. We acknowledge that a different sample in a different location may yield a different perspective on the programme. We also acknowledge that at times, the participants may have given the 'socially acceptable' answer rather than expose themselves to embarrassment in a social group.

Despite these methodological limitations, we feel that our finding offer new insights that will be beneficial for policy makers, counsellors and GPs who give advice to patients considering whether or not to complete the CRC screening test.

\section{Acknowledgements}

This study was part of a programme of work of the National Institute for Health Research Collaboration for Leadership in Applied Health Research and Care, Leicestershire, Northamptonshire and Rutland (NIHR- CLAHRC - LNR). The views expressed in this paper do not necessarily reflect those of the NIHR or the Department of Health.

The authors acknowledge financial support from Cancer Research UK and the National Cancer Action Team made available through the East Midlands Cancer Network.

Conflicts of interest

There are no conflicts of interest.

\section{References}

Armstrong D (2012). Screening: mapping medicine's temporal spaces. Sociol Health IIIn 34:177193.

Beeker C, Kraft J, Southwell B, Jorgensen C (2000). Colorectal cancer screening in older men and women: qualitative research findings and implications for intervention. $J$ Community Health 25:263-278.

Cancer Research UK (2010). Bowel cancer statistics. Available at: http:// www.cancerresearchuk.org/cancerinfo/cancerstats/types/bowel/. [Accessed 1 November 2012].

Chapple A, Ziebland S, Hewitson P, McPherson A (2008). What affects the uptake of screening for bowel cancer using a faecal occult blood test (FOBt): a qualitative study. Soc Sci Med 66:2425-2435.

Cracknell R (2010). The ageing population. Key issues for the new parliament. House of Commons Library Research. Available at: http://www.parliament.uk/ documents/commons/lib/research/key_issues/Key\%20lssues\%20The\% 20ageing\%20population2007.pdf. [Accessed 2 September 2012].

Damery S, Clifford S, Wilson S (2010). Colorectal cancer screening using the faecal occult blood test (FOBt): a survey of GP attitudes and practices in the UK. BMC Fam Pract 11:20.

Department of Health (2007). Cancer reform strategy. London: Department of Health.

Flight IHK, Wilson Cl, Griffiths L, Myers RE (2008). Interventions for improving uptake of population-based screening for colorectal cancer using the faecal occult blood testing. Cochrane Database Syst Rev Issue 4:CD005035.

Griffiths F (1995). Women's health concerns: Is the promotion of hormone replacement therapy for prevention important to women? Fam Pract 12:54-59.

Hewitson P, Glasziou PP, Irwig L, Towler B, Watson E (2007). Screening for colorectal cancer using the faecal occult blood test, Hemoccult. Cochrane Database Syst Rev Issue 1:CD001216. 
Horlick-Jones T (2011). Understanding fear of cancer recurrence in terms of damage to 'everyday health competence'. Sociol Health IIIn 33:884-898.

Keighley M, O'Morain C, Giacosa A, Ashorn M, Burroughs A, Crespi M, et al. (2004). Public awareness of risk factors and screening for colorectal cancer in Europe. Eur $J$ Cancer Prev 13:257-262.

Kitzinger J (1994). The methodology of focus groups: the importance of interaction between research participants. Sociol Health IIIn 16:103-121.

Lupton D (1995). The imperative of health: public health and the regulated body. London: Sage.

Lupton D (1999). Risk. New York: Routledge.

O'Sullivan I, Orbell S (2004). Self-sampling in screening to reduce mortality form colorectal cancer: a qualitative exploration of the decision to complete a faecal occult blood test (FOBT). J Med Screen 11:16-22.

Rowe R, Calnan M (2006). Trust relations in health care: developing a theoretical framework for the 'new' NHS. J Health Organ Manag 20:376-396.

Slovic $P$ (2000). The perception of risk. London: Earthscan.

Szczepura A, Price C, Gumber A (2008). Breast and bowel cancer screening uptake patterns over 15 years for UK south Asian ethnic minority populations, corrected for differences in socio-demographic characteristics. BMC Public Health 8:346.

Thompson T, Reeder T, Abel G (2011). 'I can't get my husband to go and have a colonoscopy': gender and screening for colorectal cancer. Health 16:235-249.

Tulchinsky T, Varavikova E (2010). What is the 'New Public Health?'. Public Health Rev 32:2553.

Wackerbarth S, Peters J, Haist S (2005). 'Do we really need all that equipment?' Factors influencing colorectal cancer screening decisions. Qual Health Res 15:539-554.

Weller D, Coleman D, Robertson R, Butler P, Campbell C, Parker R, et al. (2007). The UK colorectal cancer screening pilot: results of the second round of screening in England. $\mathrm{Br} J$ Cancer 97:1601-1605.

West N, Poullis A, Leicester R (2007). The NHS bowel cancer screening programme - a realistic approach with additional benefits. Colorectal Dis 10:708-714. 